\title{
Eddy Heat Flux in the Southern Ocean: Response to Variable Wind Forcing
}

\author{
ANDREw McC. HogG \\ Australian National University, Canberra, Australia \\ Michael P. MeRedith \\ British Antarctic Survey, Cambridge, United Kingdom \\ JEFFREY R. BLUNDELL \\ National Oceanography Centre, Southampton, Southhampton, United Kingdom \\ CHRIS WILSON \\ Proudman Oceanographic Laboratory, Liverpool, United Kingdom
}

(Manuscript received 1 March 2007, in final form 27 June 2007)

\begin{abstract}
The authors assess the role of time-dependent eddy variability in the Antarctic Circumpolar Current (ACC) in influencing warming of the Southern Ocean. For this, an eddy-resolving quasigeostrophic model of the wind-driven circulation is used, and the response of circumpolar transport, eddy kinetic energy, and eddy heat transport to changes in winds is quantified. On interannual time scales, the model exhibits the behavior of an "eddy saturated" ocean state, where increases in wind stress do not significantly change the circumpolar transport, but instead enhance the eddy field. This is in accord with previous dynamical arguments, and a recent observational study.

The instantaneous response to increased wind stress is to cool temperatures through increased northward Ekman transport of cool water. But, in the longer term, the enhanced eddy state is more efficient at transporting heat, leading to a warming of the ocean. The total eddy heat flux response is greater than the Ekman transport heat flux in this model by a factor of 2, indicating that coarse (non eddy resolving) models may fail to adequately capture the key processes. The authors also test the model response to long-term changes in wind forcing, including steadily increasing circumpolar wind strength over a 30 -yr period. The model shows a response in eddy heat flux, and a change in ocean temperature not dissimilar from observed Southern Ocean warming. These findings suggest that eddy heat flux, energized by increasing wind stress, may be a significant contributor to the observed warming of the Southern Ocean.
\end{abstract}

\section{Introduction}

The Antarctic Circumpolar Current (ACC) is a dynamically unique current encircling the Antarctic continent (Rintoul et al. 2001). The ACC plays a vital role in communicating water masses between the three major ocean basins, as well as controlling the oceanic poleward heat flux in the Southern Hemisphere. The primary mechanisms for heat transport in the surface wa-

Corresponding author address: Andrew McC. Hogg, Research School of Earth Sciences, The Australian National University, Canberra ACT 0200, Australia.

E-mail: andy.hogg@anu.edu.au ters are eddy heat flux and ageostrophic Ekman transport, since there is no mean geostrophic flow across the circumpolar streamlines that pass through Drake Passage. It follows that eddy dynamics in the Southern Ocean are critical to climate prediction, a fundamental problem for the current generation of climate models.

Satellite observations of eddy activity in the Southern Ocean indicate the presence of a significant peak in eddy kinetic energy (EKE) during 2000-02. Meredith and Hogg (2006) showed that this EKE peak lags a peak in the strength of circumpolar westerly winds [characterized by the Southern Annular Mode (SAM); Thompson and Solomon 2002] by approximately 2 yr.

DOI: 10.1175/2007JCLI1925.1 
Given the dominant role of wind forcing in the Southern Ocean, wind appears to be the most likely energy source for the anomalously large EKE. Meredith and Hogg (2006) tested this hypothesis with an eddy-resolving quasigeostrophic (QG) model of the ACC and demonstrated that a lag between wind stress forcing and EKE is likely if the system is in an "eddy saturated" state (see below for details). The lag is due to feedbacks between the mean flow, its stability and the eddies which result from instability (see also Hogg and Blundell 2006).

There are several possible implications which can be derived from the results of Meredith and Hogg (2006). First, when EKE is high, it appears likely that polewards ocean eddy heat flux will be enhanced. Second, observational records indicate that wind stress that forces the ACC is increasing (Thompson and Solomon 2002), implying that EKE in the Southern Ocean may be higher now than in the last $30 \mathrm{yr}$. Finally, the combination of these two leads to the postulation that increases in poleward eddy heat flux, driven by enhanced wind stress forcing, may be the root cause of observed warming in the Southern Ocean. Gille (2002) demonstrated that this warming is occurring from around 800to $1100-\mathrm{m}$ depth; furthermore a recent extension to this work has shown that the warming extends higher in the water column, reaching to the near-surface layers with undiminished magnitude (Gille 2008, manuscript submitted to $J$. Climate). These three possibilities are investigated in this paper using an eddy-resolving numerical model and prescribed wind stress forcing.

A number of recent modeling studies have investigated the Southern Ocean response to variable wind stress forcing, including the sea surface temperature (SST) evolution and possible coupled effects. For example, there have been several attempts to explain the observations of the Antarctic Circumpolar Wave as a coupled feedback between wind stress variability and the SST response (via ocean transport; Qiu and Jin 1997; Baines and Cai 2000). Most recently, Maze et al. (2006) used an idealized numerical model to examine this issue, concluding that the meridional heat flux due to the Ekman transport response to wind perturbations may sustain a coupled interaction.

Gnanadesikan and Hallberg (2000) used a coarseresolution $\left(3.75^{\circ} \times 4.5^{\circ}\right)$ oceanic general circulation model to investigate the oceanic response to enhanced wind stress, and observed a strong northward Ekman response which led to a centennial response in the ACC transport through stratification changes. The enhanced northward Ekman response was also observed by Oke and England (2004), who used the Bryan-Cox ocean circulation model with $1.8^{\circ} \times 3.6^{\circ}$ resolution to deter- mine the response of the ACC to a southward shift in the westerly winds. They found that the ACC migrates to the south with the wind, and that the SST evolution is dominated by increased northward Ekman transport. The Ekman response is consistent with several other studies, including Fyfe and Saenko (2005) and Saenko et al. (2005), who used global coupled climate models (at $1.85^{\circ}$ resolution) to predict that the wind stress over the Southern Ocean will both strengthen and shift southward in response to greenhouse gas forcing. The ocean circulation in these models responds in a linear fashion with a stronger, narrower and more southerly ACC. This linear response is exactly what can be expected from the residual-mean theory of the ACC (Marshall and Radko 2003). Changes in wind stress may also affect the Ekman transport and pumping (Saenko et al. 2005; Sen Gupta and England 2006), which provides a mechanism to alter the meridional overturning circulation in the region, thereby changing SSTs.

Fyfe et al. (2007) incorporate the poleward eddy heat flux into the turbulent parameterization scheme of a coarse-resolution coupled climate model, and demonstrate the possibility that enhanced mesoscale activity may increase heat flux. However, their parameterization, which involves increasing the (otherwise constant) turbulent diffusion coefficient, is relatively crude, and cannot effectively reproduce the lag in eddy response observed by Meredith and Hogg (2006).

There are a number of studies predicting that the response of the ACC to wind stress forcing is not linear. Straub (1993) proposed that, provided the wind stress was sufficiently strong, changes in wind stress would not alter the transport. Instead, transport should be controlled by stratification. This behavior is observed in a number of different eddy-resolving models (including Hallberg and Gnanadesikan 2001; Hogg and Blundell 2006; Meredith and Hogg 2006), suggesting that the dynamics of eddies, which are not necessarily well represented by eddy closure schemes, may need to be explicitly modeled to gain an accurate response. However, these eddy-resolving models are adiabatic in the sense that they specify the stratification, meaning that eddies cannot interact with the stratification, and thus will miss the thermodynamic effect on ACC transport proposed by Gnanadesikan and Hallberg (2000). This diabatic interaction is identified as being critical in the residual-mean solution of Marshall and Radko (2003).

Hallberg and Gnanadesikan (2006) address this issue by modeling the ACC using the Hallberg Isopycnic Model (HIM) at a range of resolutions from coarse $\left(2^{\circ}\right)$ to eddy permitting $\left(1 / 6^{\circ}\right)$. The latter case can simulate the diabatic flow as well as the eddy dynamics, and 
results from this model are closer to the eddy-saturated state predicted by adiabatic, eddy-resolving models. This result implies that current coarse-resolution climate models may not be able to accurately simulate the oceanic response to wind stress changes.

There is currently no clear consensus in the ocean modeling community as to whether the ACC can be adequately described by coarse-resolution models with a diffusive eddy parameterization. However, the observational record (Meredith et al. 2004; Meredith and Hogg 2006) indicates that the real ACC shows only a small change in transport in response to changing winds (maximum 5\% on interannual time scales; less than this on longer time scales), while EKE changes are spatially variable but generally larger than transport changes. Thus, there is sufficient motivation to extend the results of Meredith and Hogg (2006) to determine the response of an eddy-resolving adiabatic ocean model to changes in wind stress. We do this using a quasigeostrophic coupled model (Q-GCM) with an active mixed layer, allowing simulation of the SST and heat flux response to wind stress changes. We compare the Ekman transport heat flux with the poleward eddy heat flux to assess the relative impacts of these effects, and monitor the integrated SST field as a function of wind stress changes.

\section{Model}

We use the ocean component of the Q-GCM (version 1.3.1). The basic architecture and dynamics of the model are outlined in Hogg and Blundell (2006); see Hogg et al. (2003a,b) for a detailed account of the full coupled model.

In this model, three QG ocean layers are employed. There are three principal reasons for choosing a threelayer model. First, we (and other modelers) have observed that two-layer models inhibit eddy production via the baroclinic instability mechanism. Second, a three-layer model is the simplest that will capture modal interactions correctly [see Dewar and Morris (2000), who explicitly advocate the use of three-layer models]. Third, Maharaj et al. (2007) have shown that, at least in the South Pacific, two baroclinic modes suffice to explain almost all the observed variability of sea surface height. They see hardly any third mode, which would in any case be very poorly resolved on the $10-\mathrm{km}$ grid used in this study.

The QG equations are written in terms of the potential vorticity, $\mathbf{q}$,

$$
f_{0} \mathbf{q}-f_{0} \beta\left(y-y_{0}\right)=\nabla_{H}^{2} \mathbf{p}-f_{0}^{2} \mathbf{A} \mathbf{p}-f_{0} \tilde{\mathbf{D}},
$$

and evolves as

$$
\mathbf{q}_{t}=\frac{1}{f_{0}} J(\mathbf{q}, \mathbf{p})+\mathbf{B e}+\frac{A_{2}}{f_{0}} \nabla_{H}^{4} \mathbf{p}-\frac{A^{4}}{f_{0}} \nabla_{H}^{6} \mathbf{p},
$$

where $\mathbf{p}$ is a three-element dynamic pressure vector (one element for each layer), $f_{0}$ is the Coriolis parameter at central latitude (where $y=y_{0}$ ), $\beta$ is the (assumed constant) meridional gradient of $f_{0}, A_{2}$ and $A_{4}$ are the Laplacian and biharmonic viscosity coefficients, and $\tilde{\mathbf{D}}$ is the dynamic topography. There are two matrices in these equations: $\mathbf{A}$, which defines the relation between interface displacement and layer pressures,

$$
\mathbf{A}=\left[\begin{array}{ccc}
\frac{1}{H_{1} g_{1}^{\prime}} & \frac{-1}{H_{1} g_{1}^{\prime}} & 0 \\
\frac{-1}{H_{2} g_{1}^{\prime}} & \frac{1}{H_{2}}\left(\frac{1}{g_{2}^{\prime}}+\frac{1}{g_{1}^{\prime}}\right) & \frac{-1}{H_{2} g_{2}^{\prime}} \\
0 & \frac{-1}{H_{3} g_{2}^{\prime}} & \frac{1}{H_{3} g_{2}^{\prime}}
\end{array}\right],
$$

and $\mathbf{B}$, a $3 \times 4$ matrix that defines the forcing on the top and bottom of each layer,

$$
\mathbf{B}=f_{0}\left[\begin{array}{cccc}
\frac{1}{H_{1}} & \frac{-1}{H_{1}} & 0 & 0 \\
0 & \frac{1}{H_{2}} & \frac{-1}{H_{2}} & 0 \\
0 & 0 & \frac{1}{H_{3}} & \frac{-1}{H_{3}}
\end{array}\right] \text {, }
$$

where $H_{k}$ is the unperturbed thickness of layer $k$ and $g_{k}^{\prime}$ the reduced gravity between layers $k$ and $k+1$, with $k$ increasing downward. In addition, there is the forcing vector $\mathbf{e}$, which is given by

$$
\mathbf{e}=\left[\begin{array}{c}
w_{\mathrm{ek}} \\
0 \\
0 \\
\frac{\delta_{\mathrm{ek}}}{2\left|f_{0}\right|} \nabla_{H}^{2} p_{3}
\end{array}\right] .
$$

Here $w_{\mathrm{ek}}$ is the Ekman velocity (proportional to the wind stress curl) and the forcing also includes a linear drag on the bottom layer.

The boundary conditions in the model are applied to the pressure field. Pressure on the zonal boundaries is given by mass and momentum constraints, after McWilliams (1977). Boundary conditions are also required for the derivatives of pressure on all solid boundaries, and we use a mixed condition, following Haidvogel et al. (1992), with a nondimensional coefficient, $\alpha_{\mathrm{bc}}$, set to approximate no-slip boundary conditions. The east and 
west boundaries are periodic, so that the ocean forms a channel, mimicking the Southern Ocean.

The model includes a rudimentary mixed layer of uniform depth $H_{m}$ with varying temperature $T_{m}$. The mixed layer was designed to allow coupling between the ocean and atmosphere components for the coupled model, but in this case is used to track the evolution of SST as a function of time. This temperature evolution is calculated as

$$
\begin{aligned}
T_{m t}= & -u_{m} T_{m x}-v_{m} T_{m y}+K_{2} \nabla_{H}^{2} T_{m}-K_{4} \nabla_{H}^{4} T_{m} \\
& +\frac{F}{\rho C_{p} H_{m}},
\end{aligned}
$$

where $\left(u_{m}, v_{m}\right)$ is the mixed layer velocity, and the mixed layer heat flux is given by $F(y)=F_{0}\left(y-y_{0}\right) / Y$ and is steady for these experiments. Here $K_{2}$ and $K_{4}$ are respectively Laplacian and biharmonic SST diffusion coefficients; the latter is employed purely for numerical stability. The mixed layer boundary conditions are zero heat flux on the southern boundary and constant temperature conditions on the northern boundary. Note that these equations are a reduced form of the equations used in the full coupled version of Q-GCM (Hogg et al. 2003b); the mixed layer thus allows variation in the horizontal transport of heat, but does not adjust to increase flux to the atmosphere in the way that a fully coupled model will, nor does it allow vertical exchange with the ocean below.

The model is configured to match conditions in the Southern Ocean as closely as possible, given the idealized nature of this study. Therefore, while we are unable to include the shape of the coastlines along the Southern Ocean, we prescribe topography, derived from actual topography. The topography is obtained by averaging onto the model grid the 2-minute gridded elevations/bathymetry for the world (ETOPO2) dataset (more information is available online at http://www. ngdc.noaa.gov/mgg/fliers/01mgg04.html), which for the latitude range we consider is essentially the data of Smith and Sandwell (1997). Topographic variation greater than $O\left(\right.$ Ro $\left.\times H_{3}\right) \sim 900 \mathrm{~m}$, where Ro is the Rossby number, violates the QG assumptions, so we truncate the topography, replacing extreme values by plateaus or flat-bottomed abyssal regions. The final topography is shown in Fig. 1a.

The Ekman velocity in (5) is prescribed using

$$
w_{\mathrm{ek}}=w_{0} \sin \left(\frac{2 \pi y}{Y}\right) \text {. }
$$

The maximum dynamical wind stress (i.e., stress divided by density) corresponding to the maximum Ekman velocity in the steady case $\left(w_{0}=1.25 \times 10^{-6} \mathrm{~m} \mathrm{~s}^{-1}\right)$ is $\tau^{x} \approx 1.4 \times 10^{-4} \mathrm{~m}^{2} \mathrm{~s}^{-2}$ in the central latitudes, which is close to observed values [e.g., the Southampton Oceanography Centre (SOC) climatology of Josey et al. (2002) gives a zonal average of $\tau^{x}=1.2 \times 10^{-4}$ $\mathrm{m}^{2} \mathrm{~s}^{-2}$ for the Southern Ocean].

Table 1 shows the standard parameter values for the simulations. The parameters are designed to match Southern Ocean values as far as possible; however, we note that our Rossby radii are larger than generally accepted values for the Southern Ocean (see Houry et al. 1987), a common practice in such models. Moreover, the optimal values for viscosity coefficients are unclear-we use low viscosity under the assumption that the lowest viscosity simulations (with an active eddy field) are the closest approximation to reality.

There are two different sets of experiments. In the first, the model is spun up from rest to a statistically steady state, and winds are then temporally perturbed according to a half cosine for two years-these experiments are referred to as the perturbation experiments. This is designed to replicate the 1998-99 peak in wind stress, and uses the same forcing fields as Meredith and Hogg (2006; see Fig. 2a). The model is then run for a further $8 \mathrm{yr}$ with steady forcing to determine delayed effects to the perturbation. Two cases are run-one with a perturbation similar to observed (the standard case) and one with a larger (double) perturbation. An ensemble of 12 simulations is performed for each case to minimize the signal from internal variability. The starting condition of the ensemble members is determined by snapshots from the 120-yr steady simulation, spaced 10 yr apart.

The second group of experiments are referred to as the climatological experiments. These involve 30-yr simulations - of which the first $26 \mathrm{yr}$ have variable wind stress magnitude, and the last $4 \mathrm{yr}$ are steady. Four cases are run here: a steady reference case, a case with a linear trend matching observations, a case with just the interannual variability but no trend, and a "total" case, which is forced by both the trend and the interannual variability. Four ensemble simulations are performed for each case.

\section{Results: Perturbation experiments}

\section{a. Mean flow}

The mean state of the standard configuration for this model is shown in Figs. 1b-d. The mean SST field (Fig. 1b) shows a difference of $14^{\circ} \mathrm{C}$ across the channel, with temperature gradients smaller in the southern half of the domain. Multiple jets can be identified in the mean field. The total temperature difference, weaker gradients in the south of the domain, and multiple jets are all 


\section{(a) Topography (m)}

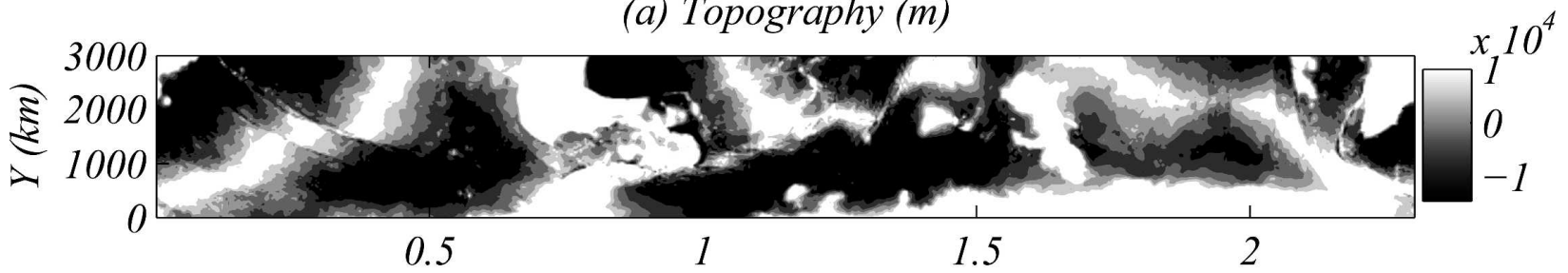

(b) $S S T$

$$
x 10^{4}
$$

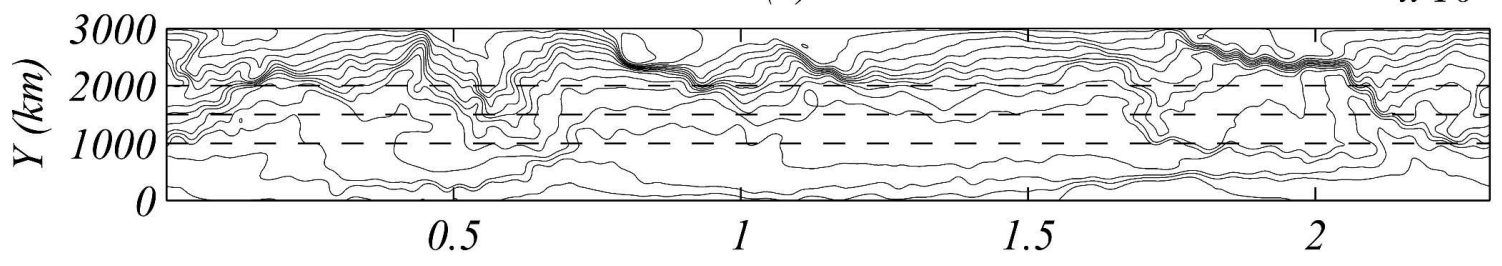

(c) $\psi_{1}$

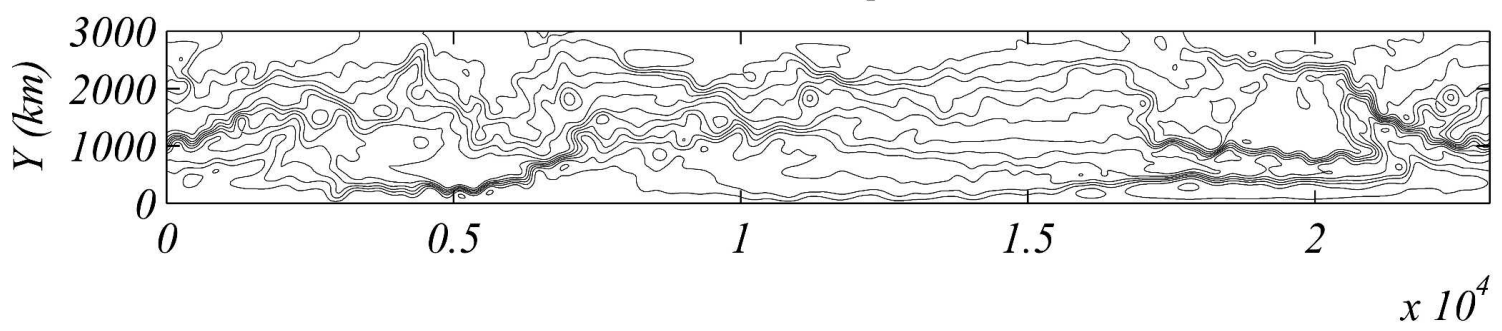

(d) $\psi_{3}$

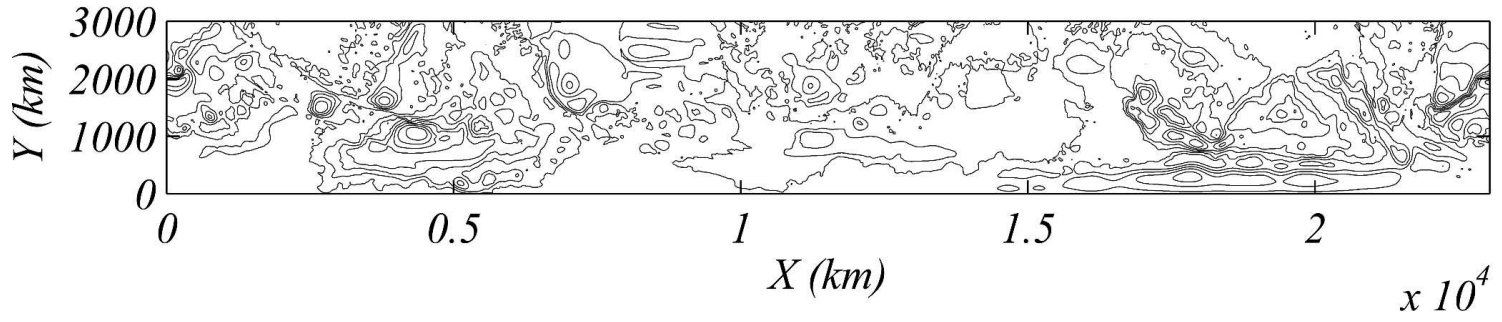

FIG. 1. (a) Model topography, (b) mean sea surface temperature field [contour interval (CI) $1^{\circ} \mathrm{C}$ ], (c) mean upper-layer streamfunction (CI $5 \mathrm{~Sv}$ ), and (d) mean lower-layer circulation (CI $10 \mathrm{~Sv}$ ). The dashed boxes in (b) indicate the areas used for averaging SST.

consistent with observations of the mean state of the ACC (Rintoul et al. 2001).

Multiple jets can be more easily identified in the mean upper-layer streamfunction (Fig. 1c). Here we see that the jets appear to be largely constrained by topography, and that 1,2 , or 3 jets may be present at any given longitude. In addition to the jets there are a number of standing eddies that can extend to the lower layer (Fig. 1d). These standing eddies play an important role in the dynamical balance of the ACC (Wolff et al. 1991) and are also topographically constrained. The total circulation of $185 \mathrm{~Sv}\left(1 \mathrm{~Sv} \equiv 10^{6} \mathrm{~m}^{3} \mathrm{~s}^{-1}\right)$ is $30 \%-$ $40 \%$ greater than observed. This transport is a strong function of stratification imposed in the model; but, given the omission of full-depth topography in this model, this can be considered to be a reasonable mean circulation. Additional simulations, performed with weaker stratification (not shown) have reduced circulation in proportion to the stratification. This result confirms the predictions of Straub (1993) that the ACC circulation is proportional to stratification; however it must be stressed that stratification in the present simulations is imposed, and cannot respond to dynamical forcing, so that one mechanism of response to different wind stress forcing is eliminated.

\section{b. Circulation response}

Figures $2 \mathrm{~b}-\mathrm{d}$ shows the domain-averaged response of key quantities to the perturbations in wind forcing for an ensemble of 12 simulations, with the ensemble 
TABLE 1. Standard parameter list for simulations, separated into fixed parameters, those varied in experiments described here, and derived parameters.

\begin{tabular}{|c|c|c|}
\hline Parameters & Value & Description \\
\hline$f_{0}$ & $-1.2 \times 10^{-4} \mathrm{~s}^{-1}$ & Mean Coriolis parameter \\
\hline$\beta$ & $1.3 \times 10^{-11}(\mathrm{~ms})^{-1}$ & Coriolis parameter gradient \\
\hline$X \times Y$ & $23040 \times 3000 \mathrm{~km}$ & Domain size \\
\hline$\Delta x$ & $10 \mathrm{~km}$ & Horizontal grid spacing \\
\hline$\Delta t$ & $15 \mathrm{~min}$ & Time step \\
\hline$\rho$ & $1000 \mathrm{~kg} \mathrm{~m}^{-3}$ & Density \\
\hline$C_{p}$ & $4000 \mathrm{~J} \mathrm{~kg}^{-1} \mathrm{~K}^{-1}$ & Specific heat capacity \\
\hline$\alpha_{\mathrm{bc}}$ & 5 & $\begin{array}{l}\text { Mixed boundary condition } \\
\text { coefficient }\end{array}$ \\
\hline$H_{k}$ & $(300,1100,2600) \mathrm{m}$ & Layer thicknesses \\
\hline$H_{m}$ & $100 \mathrm{~m}$ & Mixed layer thickness \\
\hline$g_{k}^{\prime m}$ & $(0.050,0.025) \mathrm{m} \mathrm{s}^{-2}$ & Reduced gravity \\
\hline$\delta_{\mathrm{ek}}$ & $2 \mathrm{~m}$ & $\begin{array}{l}\text { Bottom Ekman layer } \\
\text { thickness }\end{array}$ \\
\hline$A_{2}$ & $0 \mathrm{~m}^{2} \mathrm{~s}^{-1}$ & $\begin{array}{l}\text { Laplacian viscosity } \\
\text { coefficient }\end{array}$ \\
\hline$A_{4}$ & $3.0 \times 10^{10} \mathrm{~m}^{4} \mathrm{~s}^{-1}$ & $\begin{array}{l}\text { Biharmonic viscosity } \\
\text { coefficient }\end{array}$ \\
\hline$K_{2}$ & $0 \mathrm{~m}^{2} \mathrm{~s}^{-1}$ & Laplacian SST coefficient \\
\hline$K_{4}$ & $1.0 \times 10^{11} \mathrm{~m}^{4} \mathrm{~s}^{-1}$ & Biharmonic SST coefficient \\
\hline$w_{0}$ & $1.25 \times 10^{-6} \mathrm{~m} \mathrm{~s}^{-1}$ & $\begin{array}{l}\text { Amplitude of maximum } \\
\text { Ekman velocity }\end{array}$ \\
\hline \multirow[t]{4}{*}{$F_{0}$} & $40 \mathrm{~W} \mathrm{~m}^{-2}$ & $\begin{array}{l}\text { Amplitude of heat flux } \\
\text { forcing }\end{array}$ \\
\hline & $(43,26) \mathrm{km}$ & Baroclinic Rossby radii \\
\hline & 250 days & $\begin{array}{l}\text { Spindown time scale due to } \\
\text { bottom drag }\end{array}$ \\
\hline & 3.7 days & $\begin{array}{l}\text { Viscous eddy damping time } \\
\text { scale }\end{array}$ \\
\hline
\end{tabular}

spread for the steady case shown by the gray band representing \pm 1 standard deviation. The two perturbation cases have been tested for statistical significance (to the $95 \%$ confidence level) using Student's $t$ test; times at which there are significant differences from the steady case are delineated by a heavy line. There is a clear peak in potential energy (Fig. 2b) which lags the wind stress forcing maximum by less than a year. The peak is statistically significant, and exceeds the steady ensemble spread for both cases (albeit for only short periods in the perturbed case). Kinetic energy (Fig. 2c) peaks with a longer lag of 1-2 yr and shows significant differences from the standard case, while the zonal ocean transport response, Fig. 2d, shows that changes in transport are not significant, indicating that this simulation is indeed in an eddy-saturated parameter regime. These features are consistent with the results of Meredith and Hogg (2006).

\section{c. SST response}

The numerical simulations performed here are designed to allow calculation of the temperature evolu-
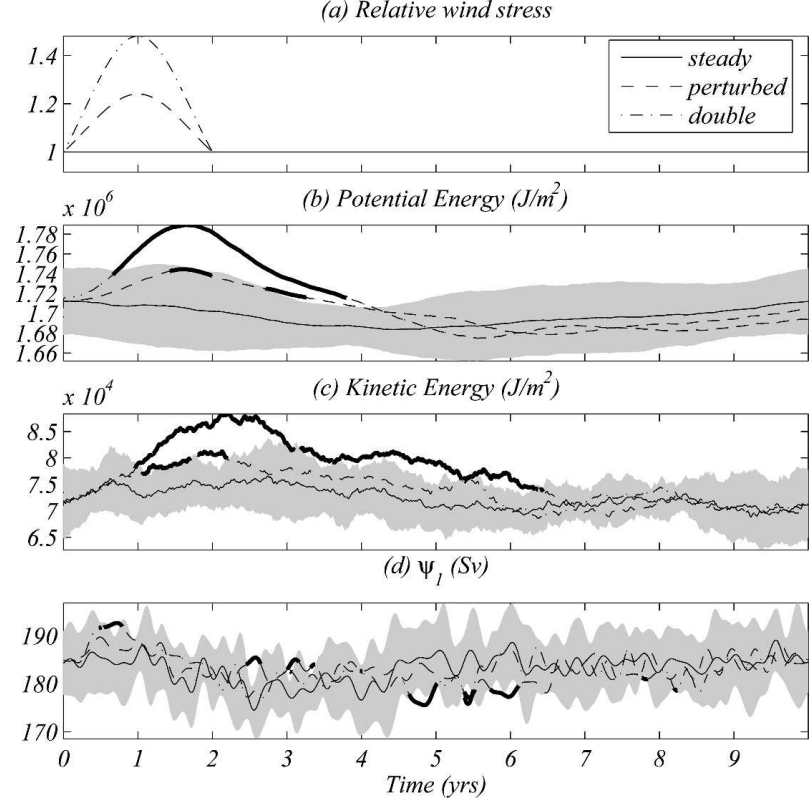

FIG. 2. Time series of (a) relative wind stress forcing, (b) domain-averaged potential energy, (c) domain-averaged kinetic energy, and (d) upper-layer streamfunction for an ensemble of 12 simulations. The gray band shows the ensemble spread for the steady case, while thick lines delineate times at which differences are statistically significant.

tion in the channel. SST is averaged over two regions south and north of the ACC to enable a simple time series diagnosis of the SST evolution. There are a number of ways in which the temperature record may be averaged to produce a time series. One possible method, mimicking techniques often used for Southern Ocean observations, is to use mean streamlines to define regions, and average temperature over these two regions. An alternative method is to simply average over latitude bands. We have tested both of these methods and find no qualitative differences between the results, and therefore elect to use the simpler method of latitude bands. Two latitude bands are defined: a region south of the center of the domain, between $y=1000$ and $1500 \mathrm{~km}$, and a region north of the center between $y=1500$ and $2000 \mathrm{~km}$. These two regions are respectively south and north of the central part of the model ACC; although the current passes through both regions because of large meridional deviations (see Fig. 1).

We define the temperature difference, $\Delta T=T_{\text {north }}-$ $T_{\text {south }}$, and plot the ensemble average of this quantity as a function of time for the steady, perturbed, and double cases in Fig. 3a. The mean value of the temperature difference is relatively small, about $1.5^{\circ} \mathrm{C}$, and the ensemble spread of the steady forcing case (gray band) shows the high internal variability. There are differ- 


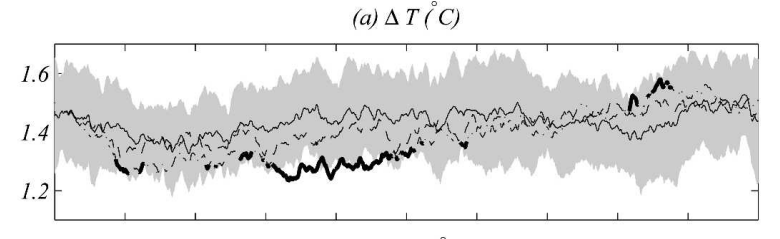

(b) $T_{\text {north }}$ ( $C$ )

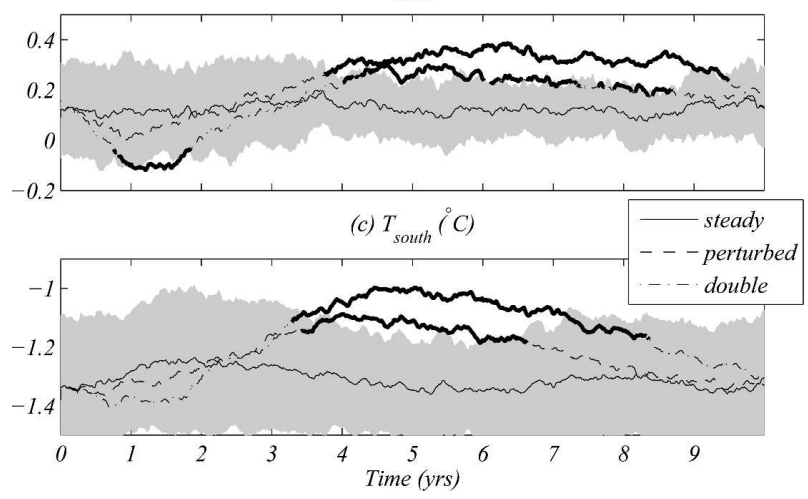

FIG. 3. Time series of (a) difference in SST between the northern and southern regions, (b) SST in the northern region, and (c) SST in the southern region from an ensemble of 12 simulations. The gray band shows the ensemble spread for the steady case, while thick lines delineate times at which differences are statistically significant.

ences between the perturbed cases and the steady case, with a temperature gradient for years $1-6$, reduced by $0.1^{\circ} \mathrm{C}$ for the perturbed case, and by $0.2^{\circ} \mathrm{C}$ for the double perturbation, but these differences are only statistically significant in the double perturbation case.

The contribution of the north and south regions to the temperature gradient changes are clarified in Figs. 3b,c where we plot $T_{\text {north }}$ and $T_{\text {south }}$, respectively (relative to the basin-averaged mean temperature). These plots show an initial cooling in both regions during the wind stress event. After the wind stress perturbation, in years $3-6$, both regions increase in temperature by $0.2^{\circ}-$ $0.4^{\circ} \mathrm{C}$, and these increases are clearly significant for both cases.

These results are consistent with the hypothesis that the first response is due to enhanced northward Ekman transport of cool water, while the delayed response is due to enhanced poleward eddy heat flux, which increases the temperature in both regions. We test this hypothesis by explicitly calculating the eddy and Ekman heat flux components for each case below.

\section{d. Heat flux calculations}

The contributions of eddy and Ekman heat flux to SST changes are diagnosed by integrating the heat flux over both the north and south regions defined above. The meridional eddy heat flux can be calculated by decomposing northward mixed layer velocity and the mixed layer temperature into a time-averaged and a perturbation component:

$$
\begin{gathered}
v_{m}=\overline{v_{m}}+v_{m}^{\prime}, \\
T_{m}=\overline{T_{m}}+T_{m}^{\prime} .
\end{gathered}
$$

The total northward heat flux across a circumpolar section is then given by

$$
\begin{aligned}
\oint_{\rho} C_{p} H_{m} v_{m} T_{m} d x= & \oint_{\rho} C_{p} H_{m} \overline{v_{m}} \overline{T_{m}} d x \\
& +\oint_{\rho} C_{p} H_{m}\left[v_{m}^{\prime} \overline{T_{m}}+\overline{v_{m}} T_{m}^{\prime}\right. \\
& \left.+v_{m}^{\prime} T_{m}^{\prime}\right] d x,
\end{aligned}
$$

where the first term on the right represents mean advective heat flux, and the terms in square brackets are the perturbation heat flux. Note that we have integrated heat flux over the (constant depth) mixed layer, and scaled with heat capacity so that heat flux has units of Watts. In some cases it would be possible to average this equation in time once again, and thereby eliminate the first two terms in the square brackets. In the case described here, where the flow is time dependent and thus not statistically steady, it is not possible to do this. We therefore include all three time-varying terms in our estimate of heat flux.

We can also decompose the mixed layer velocity into two components,

$$
v_{m}=\frac{p_{1 x}}{f_{0}}-\frac{\tau^{x}}{H_{m} f_{0}},
$$

where $p_{1}$ is the layer 1 dynamic pressure and $\tau^{x}$ the applied wind stress in the zonal direction. The first component here is the geostrophic velocity, which will include both the mean and perturbation quantities. We assume that the mean geostrophic flow is predominantly due to the large-scale circulation, while the perturbation geostrophic velocity is used as a proxy for the eddy field. The second mixed layer velocity component is the ageostrophic flow, which is the Ekman transport in the mixed layer.

The ensemble average geostrophic (Fig. 4a) and ageostrophic (Fig. 4b) heat flux anomalies are plotted in Fig. 4, averaged over a latitude band that includes both the north and south regions defined above (i.e., between $y=1000$ and $2000 \mathrm{~km}$ ). The eddy heat flux has a very significant component of noise, and the signal we observe is smaller in amplitude than the variation between ensembles, making it difficult to distinguish the excess eddy flux signal in years 3-6. Nonetheless, the 

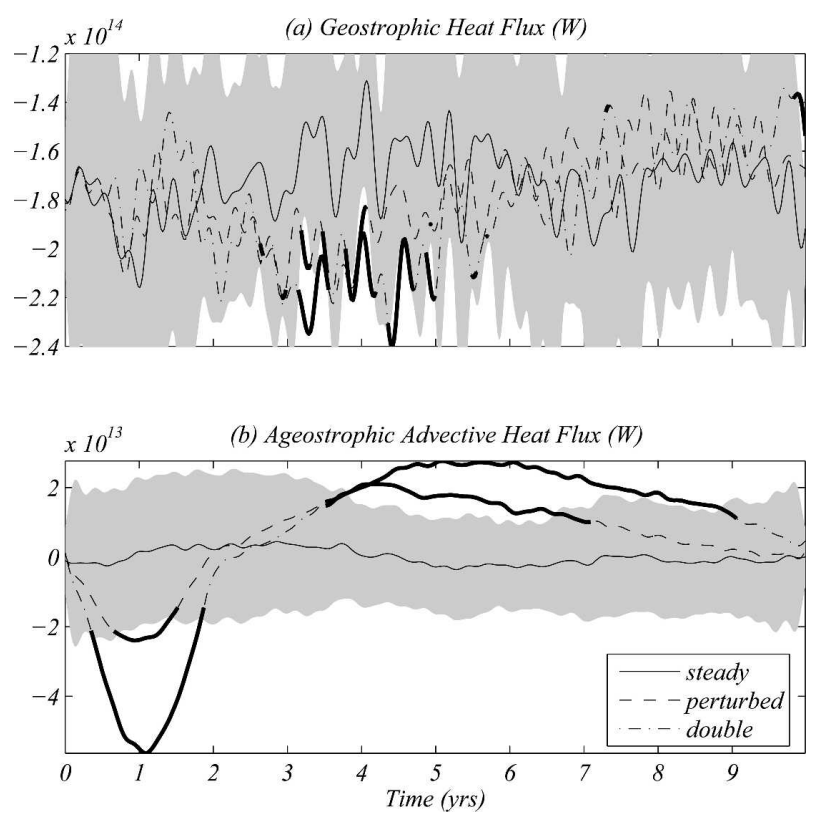

FIG. 4. Time series of (a) geostrophic heat flux, representing eddy heat flux; and (b) ageostrophic heat flux, consisting of Ekman transport. The gray band shows the ensemble spread for the steady case, while thick lines delineate times at which differences are statistically significant.

anomalous negative heat flux in years 4 and 5 is statistically significant for the double case-this is a southward flux of heat due to horizontal mixing by the eddies (consistent with their growth through baroclinic instability). An average over years 3-6 indicates that the standard and double perturbations cause heat flux anomalies of $-1.8 \times 10^{13}$ and $-3.2 \times 10^{13} \mathrm{~W}$, respectively; when averaged over this time interval the double perturbation case exceeds the ensemble standard deviation of $2.5 \times 10^{13} \mathrm{~W}$.

The Ekman heat transport shows a more systematic response, with a well-defined minimum in the Ekman transport (ageostrophic heat flux) response to the perturbation, which is directly proportional to the imposed wind forcing anomaly. The negative heat flux is consistent with a northward flux of cool water. This effect will act to reduce the temperature gradient and cool the northern region, consistent with the SST response seen in Fig. 3 for years $1-2$ of the simulation. The average heat flux is of the same magnitude as the eddy flux $\left(-1.5 \times 10^{13}\right.$ and $-3.5 \times 10^{13} \mathrm{~W}$, respectively), but only lasts for the duration of the wind perturbation, so that total Ekman heat flux is a factor of 2 smaller than the eddy heat flux. This result is in contrast to numerous coarse-resolution studies where Ekman transport dominates the SST response, and may be explained by excessive damping or underrepresentation of the eddy field in coarse models. In the present model the eddies outlast the wind perturbation and so have the potential to continue heat transfer. The Ekman transport anomalies in the latter stages of the experiment are positive, particularly in years 5-8. This effect is due to a positive temperature anomaly (relative to the mean), rather than increased velocity [i.e., it is the $-\overline{\left(\tau_{x} / H_{m} f_{0}\right)} T_{m}^{\prime}$ term]. This is a direct result of warming by poleward eddy heat flux and highlights a specific feature of this analysis: that temperature anomalies are defined relative to a long-term mean in simulations that are not statistically steady.

These results suggest that enhanced winds will reduce the north-south temperature gradient in this model because of subsequent enhanced eddy activity. Ekman flux provides an instantaneous cooling while the wind is perturbed and is a restoring influence once warming has occurred. The poleward eddy heat flux acts to warm both regions with a lag of 2-4 yr, which is consistent with expectations. However, the signal is very difficult to discern directly (despite the 12-run ensemble average and domain averaging), because the ensemble spread is so large. Therefore, even if this effect were present in the real ocean it would be almost impossible to see a signal from the satellite record. The perturbation experiments have been repeated for simulations with weaker stratification; results are consistent with the above results, so only one case is shown here.

\section{Results: Climatological experiments}

The above experiments show how, if the ACC is in an eddy-saturated state, interannual variability of the wind stress produces an increase in the EKE and poleward eddy heat flux, with a lag of several years, which is stronger than Ekman heat flux anomalies. It follows that slower variations in the wind stress field will also modify both EKE and eddy heat flux, but that the lagged response will play a smaller role. It is pertinent to note that wind stress forcing in the Southern Ocean has undergone a gradual increase over the last $30 \mathrm{yr}$ (Thompson and Solomon 2002). We now mimic these trends with the current model to examine the exact nature of this effect.

We use monthly wind stress data from the National Centers for Environmental Prediction (NCEP) reanalysis. NCEP data are available online at http://www. cdc.noaa.gov/cdc/reanalysis/reanalysis.shtml. Zonal averages of eastward wind stress were derived from these data, deseasonalized by subtracting monthly means from the respective months and then smoothed with a Butterworth filter with a cutoff at 1 cpy. Data from 
(a) Relative wind stress
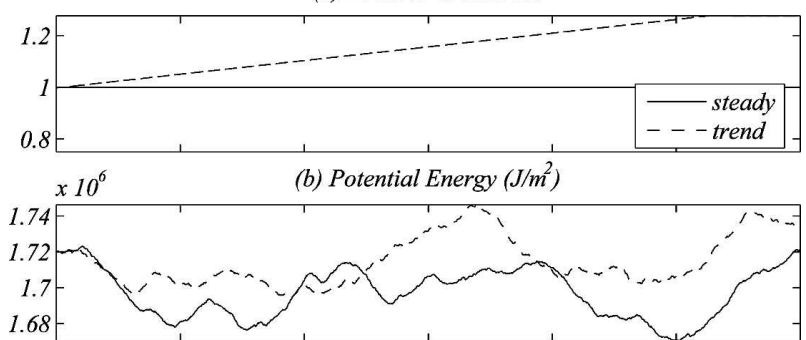

$\times 10^{4} \quad$ (c) Kinetic Energy $\left(\mathrm{J} / \mathrm{m}^{2}\right)$
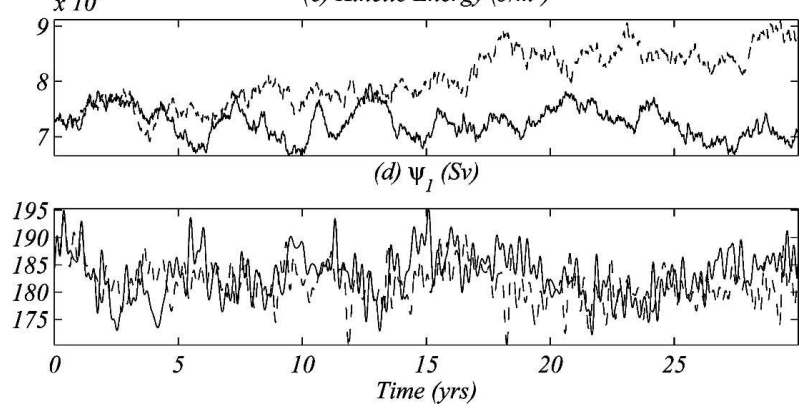

FIG. 5. Time series of (a) relative wind stress forcing, (b) domain-averaged potential energy, (c) domain-averaged kinetic energy, and (d) upper-layer streamfunction for the steady case and the case with a linear trend in wind stress. Data are the mean across 4 ensemble simulations.

$26 \mathrm{yr}$ from 1976 to 2002 is separated into two components: a linear trend and the interannual variability, with $4 \mathrm{yr}$ of steady wind after that time. Coefficients for amplification of wind stress forcing with time are extracted from this data. Four ensemble experiments of 30 -yr duration are performed. We compare these results with the steady case, and with a simulation (called total) with both the trend and interannual variability present.

\section{a. Circulation response}

Results from the linear trend forcing and steady case are compared in Fig. 5. Both simulations possess similar levels of internal variability in the potential and kinetic energy records, and it is notable that the restriction of only four ensemble simulations does not eliminate this internal variability. The mean value of potential energy differs marginally between the two cases. On the other hand, EKE is approximately $20 \%$ higher for the trend case by the end of the 30 -yr simulation, which is roughly proportional to the increase in wind energy input. The zonal transport changes little between the two cases, and is even slightly smaller for the linear trend case during the final years of the simulation.

Similar patterns are seen when the simulation with interannual variability is compared with the total forcing in Fig. 6. The interannual variability in the wind forcing sets the timing of variability in the potential and
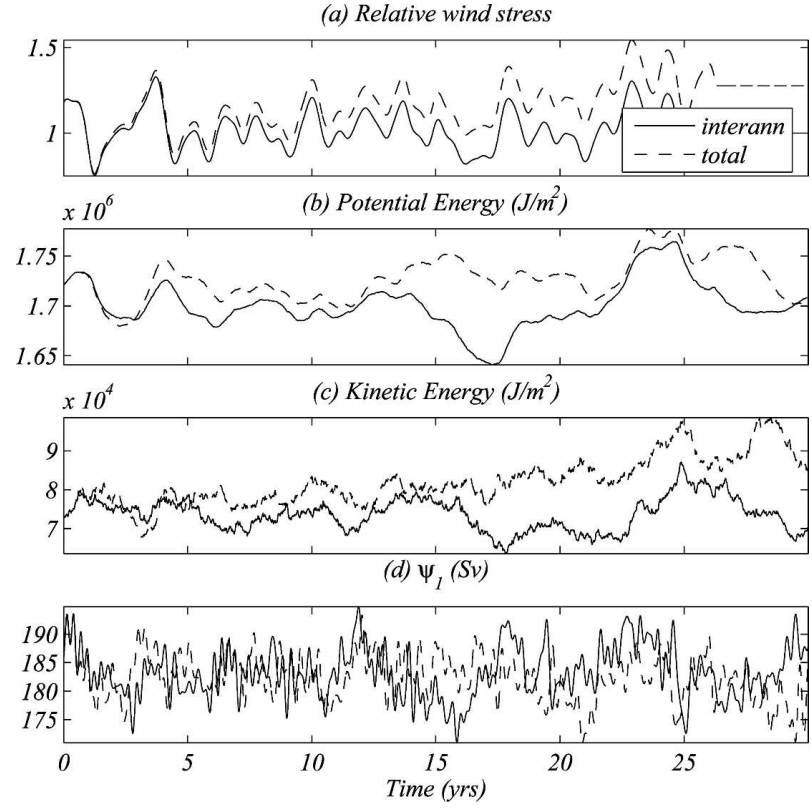

FIG. 6. Time series of (a) relative wind stress forcing, (b) domain-averaged potential energy, (c) domain-averaged kinetic energy, and (d) upper-layer streamfunction for the interannual variability case and the total forcing case. Data are the mean across 4 ensemble simulations.

kinetic energy fields, except that the total forcing case responds faster because of a higher mean EKE state (this behavior is predicted by Hogg and Blundell 2006). By the end of the 30-yr simulation, potential energy levels are similar, while EKE is higher in the total forcing case. Zonal transport is similar in each case. These results are consistent with expectations from previous simulations and imply that we can expect that eddy heat transport may be proportional to wind stress in the longer term.

\section{b. SST response}

The temperature responses for the four cases are shown in Figs. 7, 8 using the same definitions for averaging regions as Fig. 3. This data show that a decrease in temperature difference by $0.1^{\circ} \mathrm{C}$ is detectable for the trend and total cases. In addition, the total temperature in each region increases by $0.3^{\circ}-0.4^{\circ} \mathrm{C}$ when the interdecadal trend is included. The interannual temperature response for the cases that include interannual forcing (Fig. 8) is controlled by the forcing variability.

In the perturbation experiments shown in section 3, the Ekman heat flux acts to cool both regions, while the poleward eddy heat flux warms both regions. It therefore appears that the SST trends shown here are dominated by eddy heat flux. We aim to confirm this prediction with explicit calculations. 

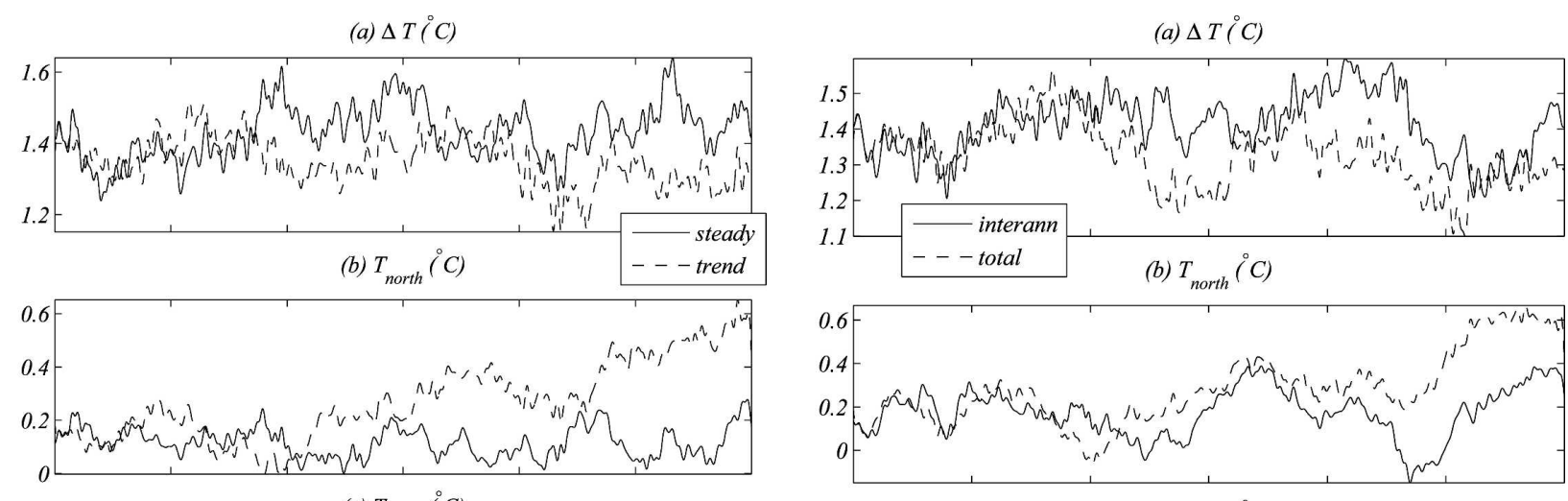

(c) $T_{\text {south }}(\mathrm{C})$

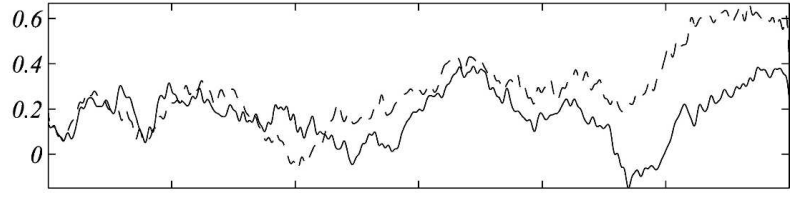

(c) $T_{\text {south }}(\mathrm{C})$

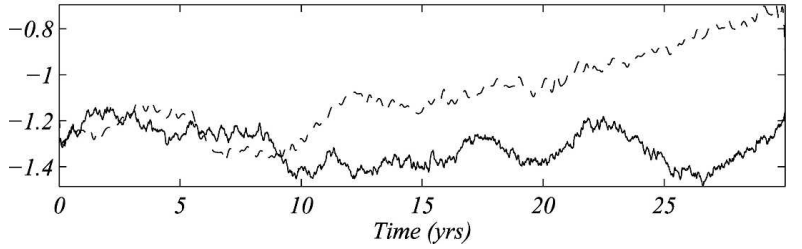

FIG. 7. Time series of (a) difference in SST between the northern and southern regions, (b) SST in the northern region, and (c) SST in the southern region for the steady case and the case with a linear trend in wind stress. Data are the mean across 4 ensemble simulations.

\section{c. Heat flux calculations}

We calculate the thermal forcing due to anomalous Ekman transport and due to eddy heat flux as per section $3 \mathrm{c}$. The comparison of the steady and trend heat fluxes (Fig. 9) show once again that internal variability obscures a clear signal. Nonetheless, there is a clear and systematic difference between the eddy heat fluxes for these two cases in the second half of the simulation. The average difference is $-11.3 \times 10^{12} \mathrm{~W}$ over this time, which is of the right sign to produce the warming trend shown in Fig. 7. However it is a factor of 3 smaller than the heat flux anomalies for the perturbation experiments-this is most likely due to the feedback caused by increasing SST during the course of the simulation. The Ekman heat fluxes are not as systematic. Initially, there is a slightly negative Ekman heat flux for cases in which the wind is amplified, consistent with a small northward enhancement to Ekman transport. In the latter stages of the simulation, this sign is reversed. The mean flux anomaly over the second half of this run is $7.7 \times 10^{12} \mathrm{~W}$ which is smaller than the eddy component and the opposite sign expected from increased northward Ekman transport (again this effect is due to positive SST anomalies in the region of interest).

Figure 10 shows that interannual variability plays a strong role in setting the heat flux, as described in section 3. However, the differences between the interan-

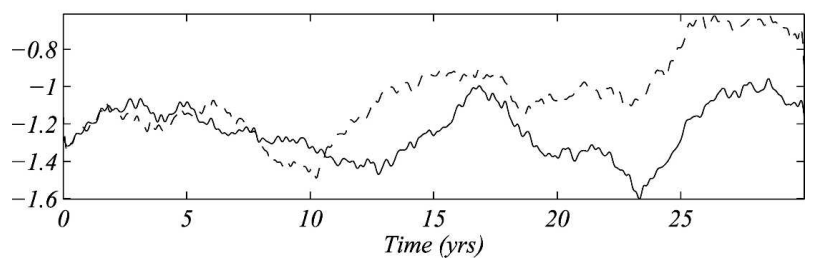

FIG. 8. Time series of (a) difference in SST between the northern and southern regions, (b) SST in the northern region, and (c) SST in the southern region for the interannual variability case and the total forcing case. Data are the mean across 4 ensemble simulations.

nual and total cases show eddy heat flux differences of $-8.5 \times 10^{12} \mathrm{~W}$, and Ekman heat flux differences of over the $6.6 \times 10^{12} \mathrm{~W}$ over the second half of the run. These values are consistent with the differences between the steady and trend cases above, and show that even with interannual variability included the eddy heat flux dominates the temperature response over the longer term.

\section{Discussion}

Fyfe and Saenko (2006) show that many global coupled climate models have an ACC that responds linearly to changing wind stress patterns in the Southern Ocean. The ACC follows the wind forcing in that the zonal transport increases in proportion to the wind and the current shifts meridionally as the wind does. This is consistent with the residual-mean model of the ACC by Marshall and Radko (2003), who use an eddy closure scheme to predict a linear dependence of circumpolar transport on wind stress. That model has the advantage that it allows for feedback between the stratification and eddy activity. On the other hand, other models of the ACC, such as Straub (1993), predict that increasing the wind stress does not change the ACC. These models generally have stratification specified, but resolve eddy dynamics. 

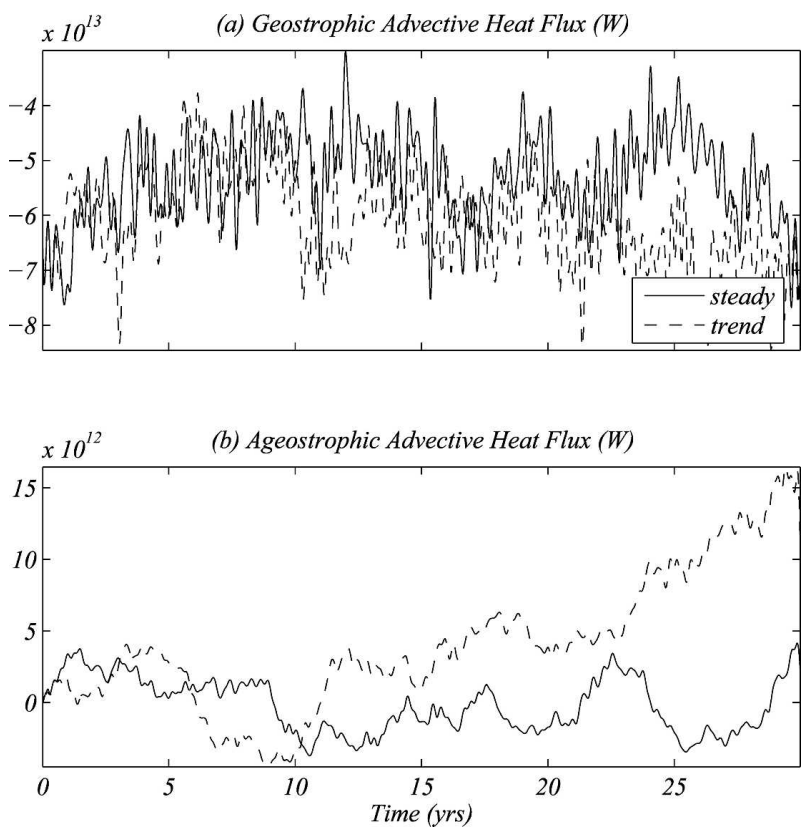

FIG. 9. Time series of (a) geostrophic heat flux, representing eddy heat flux; and (b) ageostrophic heat flux, consisting of Ekman transport for the steady case and the case with a linear trend in wind stress. Data are the mean across 4 ensemble simulations.

The results described here fall into the latter category, where the zonal circumpolar transport is in the eddy-saturated parameter regime. This study does not directly contribute to the debate on whether the ACC is in an eddy-saturated state; however, we have shown previously, on the basis of observation data, that the real ACC is very likely close to an eddy-saturated state (Meredith et al. 2004; Meredith and Hogg 2006). We therefore assume the existence of an eddy-saturated parameter regime and examine the consequences of that assumption.

When the circumpolar current is eddy saturated there is a clear decrease in the temperature gradient following periods of strong wind stress. Associated with the temperature gradient decrease is an initial cooling period due to northward Ekman transport. However, the longer-term effect is a warming of SST, which is strongest south of the ACC, lagging wind stress forcing by several years. These temperature trends are primarily due to enhanced poleward eddy heat flux, which in turn results from high EKE. The total eddy heat flux in this model is greater than the Ekman heat flux by a factor of 2 (but this result occurs because eddy effects are longer lived-instantaneous fluxes are similar in magnitude). This result could not be obtained using a standard diffusive eddy parameterization such as those used in coarse-resolution ocean and climate models.

Under a gradual increase in wind stress forcing over
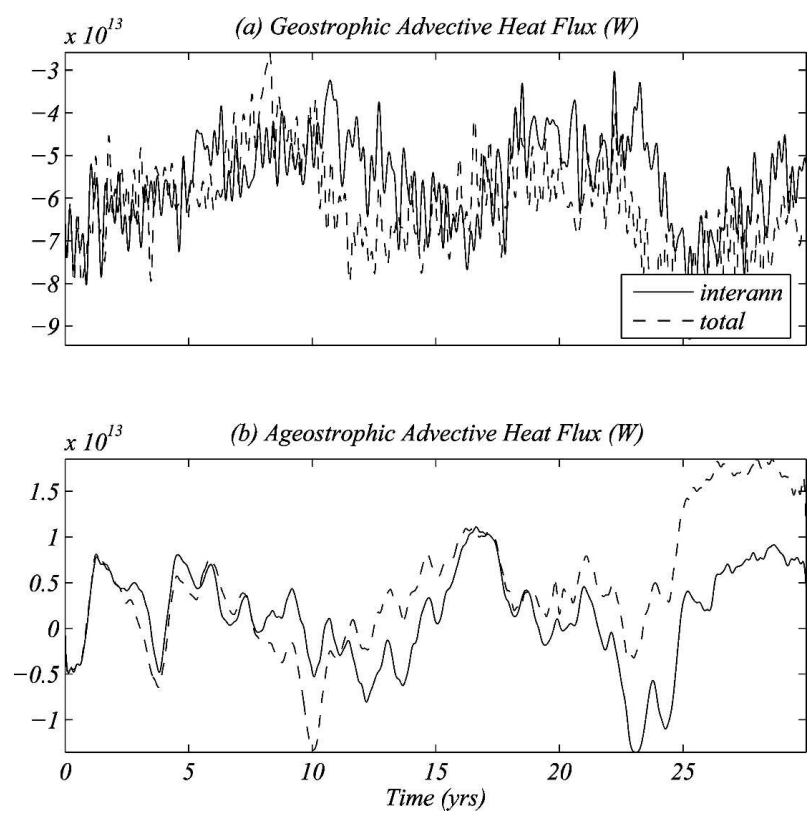

FIG. 10. Time series of (a) geostrophic heat flux, representing eddy heat flux; and (b) ageostrophic heat flux, consisting of Ekman transport for the interannual variability case and the total forcing case. Data are the mean across 4 ensemble simulations.

$30 \mathrm{yr}$ the temperature gradient also decreases. This is due to the same physical effects, except that the lag is not so important. The temperature decrease is significant for the parameters used here-approaching $0.5^{\circ} \mathrm{C}$ warming over $30 \mathrm{yr}$. This rate of warming is similar to that observed in the Southern Ocean, implying that poleward eddy heat flux may play a role in anomalous warming south of the ACC.

There are several courses of action that may help resolve the extent to which wind-forced eddy heat flux has played a role in anomalous Southern Ocean forcing. First, we might appeal to the satellite record itself, and attempt to directly calculate eddy heat flux. However, the simulations performed here demonstrate that the eddy heat flux is overwhelmed by internal variability, even over a number of ensemble simulations. It follows that any diagnosis of eddy heat fluxes from satellite observations (a single realization sampled at lower spatial resolution) will be difficult.

A second method to determine whether the dynamics seen in this model are active in the real world is to examine correlations between temperature gradients and wind stress forcing. The link between an interdecadal increase in wind strength and Southern Ocean warming exists already. However, the mechanism described here has a 3-6-yr lag in the SST response to interannual wind stress forcing variability, which may help to identify a signal. 
Third, the relevance of the results described here can be demonstrated if it can be determined that the ACC state can be considered eddy saturated. This can be achieved by examining the dynamics of eddy-resolving models that include realistic diabatic effects and evolving stratification. Hallberg and Gnanadesikan (2006) are the first to attempt this. They find that as resolution is increased "the diabatic response to changes in wind forcing is substantially attenuated by the action of a vigorous eddy field as suggested by the adiabatic view." This lends support to the hypothesis that the eddysaturated regime is relevant to the Southern Ocean circulation. The results outlined in the current paper lend a sense of urgency to resolving this debate with additional modeling studies.

There are additional potential ramifications of the results described here to the Southern Ocean climate system. Marshall and Connolley (2006) propose that winter SSTs may be altering the strength of the SAM. If true, this could imply coupled feedbacks between the poleward eddy heat flux and the pattern of the SAM. It is most likely that this would take the form of damped decadal oscillations in the coupled system. Such oscillations would be controlled by the lagged response of the eddy heat flux, and the dependence of the SAM on high-latitude SST. This may result in an extension of the coupled feedbacks proposed by Sen Gupta and England (2007). These feedbacks helped to sustain variability in the SAM, but it was noted that time scales did not match observations. This mismatch may be reconciled by the inclusion of a realistic delay in the system due to eddy effects as shown here.

\section{Conclusions}

An eddy-resolving QG model of the Southern Ocean is used to demonstrate the following:

1) Interannual perturbations to the wind stress forcing of the ACC increase SST with a lag in SST maximum 3-6 yr after the wind stress maximum;

2) Poleward eddy heat flux is a larger effect than equatorward Ekman transport;

3) A slower (30 yr) amplifying trend in wind stress acts to warm waters around the ACC, with heat flux due to eddies exceeding Ekman transport by a factor 3 .

It is proposed that eddy heat flux due to increasing wind forcing may explain observations of enhanced warming of the Southern Ocean.

Acknowledgments. This work was supported by the National Facility of the Australian Partnership for Advanced Computing. AMH was supported by an Austra- lian Research Council Postdoctoral Fellowship (DP0449851). CW was funded by the U.K. Natural Environmental Research Council under Oceans2025 Theme 1: Climate, Ocean Circulation and Sea Level.

\section{REFERENCES}

Baines, P. G., and W. Cai, 2000: Analysis of an interactive instability mechanism for the Antarctic circumpolar wave. J. Climate, 13, 1831-1844.

Dewar, W. K., and M. Y. Morris, 2000: On the propagation of baroclinic waves in the general circulation. J. Phys. Oceanogr., 30, 2637-2649.

Fyfe, J. C., and O. A. Saenko, 2005: Human-induced changes in the Antarctic Circumpolar Current. J. Climate, 18, 30683073.

— Southern Hemisphere winds and currents. Geophys. Res. Lett., 33, L06701, doi:10.1029/2005GL025332.

$\longrightarrow,-$ K. Zickfeld, M. Eby, and A. J. Weaver, 2007: The role of poleward-intensifying winds on Southern Ocean warming. J. Climate, 20, 5391-5400.

Gille, S. T., 2002: Warming of the Southern Ocean since the 1950s. Science, 295, 1275-1277.

Gnanadesikan, A., and R. Hallberg, 2000: On the relationship of the Circumpolar Current to Southern Hemisphere winds in coarse-resolution ocean models. J. Phys. Oceanogr., 30, $2013-$ 2034.

Haidvogel, D. B., J. C. McWilliams, and P. R. Gent, 1992: Boundary current separation in a quasigeostrophic, eddy-resolving ocean circulation model. J. Phys. Oceanogr., 22, 882-902.

Hallberg, R., and A. Gnanadesikan, 2001: An exploration of the role of transient eddies in determining the transport of a zonally reentrant current. J. Phys. Oceanogr., 31, 3312-3330.

— and - 2006: The role of eddies in determining the structure and response of the wind-driven Southern Hemisphere overturning: Results from the Modeling Eddies in the Southern Ocean (MESO) project. J. Phys. Oceanogr., 36, 22322252.

Hogg, A. M., and J. R. Blundell, 2006: Interdecadal variability of the Southern Ocean. J. Phys. Oceanogr., 36, 1626-1645.

, — - W. K. Dewar, and P. D. Killworth, 2003a: Formulation and users' guide for Q-GCM (version 1.0). Southampton Oceanography Centre, Internal Doc. 88, 44 pp. [Available online at http://www.noc.soton.ac.uk/JRD/PROC/Q-GCM/ q-gcmv1.0.pdf.]

— W. W. Dewar, P. D. Killworth, and J. R. Blundell, 2003b: A quasi-geostrophic coupled model (Q-GCM). Mon. Wea. Rev., 131, 2261-2278.

Houry, S., E. Dombrowsky, P. De Mey, and J.-F. Minster, 1987: Brunt-Väisälä frequency and Rossby radii in the South Atlantic. J. Phys. Oceanogr., 17, 1619-1626.

Josey, S. A., E. C. Kent, and P. K. Taylor, 2002: Wind stress forcing of the ocean in the SOC climatology: Comparisons with the NCEP-NCAR, ECMWF, UWM/COADS, and Hellerman and Rosenstein datasets. J. Phys. Oceanogr., 32, 1993 2019.

Maharaj, A. M., P. Cipollini, N. J. Holbrook, P. D. Killworth, and J. R. Blundell, 2007: An evaluation of the classical and extended Rossby wave theories in explaining spectral estimates of the first few baroclinic modes in the South Pacific Ocean. Ocean Dyn., 57, 173-187. 
Marshall, G. J., and W. M. Connolley, 2006: The effect of changing Southern Hemisphere winter sea surface temperatures on Southern Annular Mode strength. Geophys. Res. Lett., 33, L17717, doi:10.1029/2006GL026627.

Marshall, J., and T. Radko, 2003: Residual-mean solutions for the Antarctic Circumpolar Current and its associated overturning circulation. J. Phys. Oceanogr., 33, 2341-2354.

Maze, G., F. D'Andrea, and A. Colin de Verdière, 2006: Lowfrequency variability in the Southern Ocean region in a simplified coupled model. J. Geophys. Res., 111, C05010, doi:10.1029/2005JC003181.

McWilliams, J. C., 1977: A note on a consistent quasigeostrophic model in a multiply connected domain. Dyn. Atmos. Oceans, 1, 427-441.

Meredith, M. P., and A. M. Hogg, 2006: Circumpolar response of Southern Ocean eddy activity to a change in the Southern Annular Mode. Geophys. Res. Lett., 33, L16608, doi:10.1029/ 2006 GL026499.

, P. L. Woodworth, C. W. Hughes, and V. Stepanov, 2004: Changes in the ocean transport through Drake Passage during the 1980s and 1990s, forced by changes in the Southern Annular Mode. Geophys. Res. Lett., 31, L21305, doi:10.1029/ 2004 GL021169.

Oke, P. R., and M. H. England, 2004: Oceanic response to changes in the latitudes of the Southern Hemisphere subpolar westerly winds. J. Climate, 17, 1040-1054.

Qiu, B., and F.-F. Jin, 1997: Antarctic circumpolar waves: An indication of ocean-atmosphere coupling in the extratropics. Geophys. Res. Lett., 24, 2585-2588.

Rintoul, S. R., C. W. Hughes, and D. Olbers, 2001: The Antarctic Circumpolar Current system. Ocean Circulation and Climate: Observing and Modelling the Global Ocean, G. Siedler, J. Church, and J. Gould, Eds., Academic Press, 271-302.

Saenko, O. A., J. C. Fyfe, and M. H. England, 2005: On the response of the oceanic wind-driven circulation to atmospheric $\mathrm{CO}_{2}$ increase. Climate Dyn., 25, 415-426.

Sen Gupta, A., and M. H. England, 2006: Coupled oceanatmosphere-ice response to variations in the Southern Annular Mode. J. Climate, 19, 4457-4486.

- and - 2007: Coupled ocean-atmosphere feedback in the Southern Annular Mode. J. Climate, 20, 3677-3692.

Smith, W. H. F., and D. T. Sandwell, 1997: Global sea floor topography from satellite altimetry and ship depth soundings. Science, 277, 1956-1962.

Straub, D. N., 1993: On the transport and angular momentum balance of channel models of the Antarctic Circumpolar Current. J. Phys. Oceanogr., 23, 776-782.

Thompson, D. W. J., and S. Solomon, 2002: Interpretation of recent Southern Hemisphere climate change. Science, 296, 895-899.

Wolff, J.-O., E. Maier-Reimer, and D. J. Olbers, 1991: Winddriven flow over topography in a zonal $\beta$-plane channel: A quasi-geostrophic model of the Antarctic Circumpolar Current. J. Phys. Oceanogr., 21, 236-264. 